\title{
Computing the scattering properties of participating media using Lorenz-Mie theory
}

Frisvad, Jeppe Revall; Christensen, Niels Jørgen; Jensen, Henrik Wann

Published in:

ACM Transactions on Graphics

Link to article, DOI:

$10.1145 / 1276377.1276452$

Publication date:

2007

Link back to DTU Orbit

Citation (APA):

Frisvad, J. R., Christensen, N. J., \& Jensen, H. W. (2007). Computing the scattering properties of participating media using Lorenz-Mie theory. ACM Transactions on Graphics, 26(3), article number 60.

https://doi.org/10.1145/1276377.1276452

\section{General rights}

Copyright and moral rights for the publications made accessible in the public portal are retained by the authors and/or other copyright owners and it is a condition of accessing publications that users recognise and abide by the legal requirements associated with these rights.

- Users may download and print one copy of any publication from the public portal for the purpose of private study or research.

- You may not further distribute the material or use it for any profit-making activity or commercial gain

- You may freely distribute the URL identifying the publication in the public portal

If you believe that this document breaches copyright please contact us providing details, and we will remove access to the work immediately and investigate your claim. 


\title{
Computing the Scattering Properties of Participating Media Using Lorenz-Mie Theory
}

\author{
Jeppe Revall Frisvad $^{1} \quad$ Niels Jørgen Christensen ${ }^{1} \quad$ Henrik Wann Jensen ${ }^{2}$ \\ ${ }^{1}$ Informatics and Mathematical Modelling, Technical University of Denmark \\ ${ }^{2}$ University of California, San Diego
}

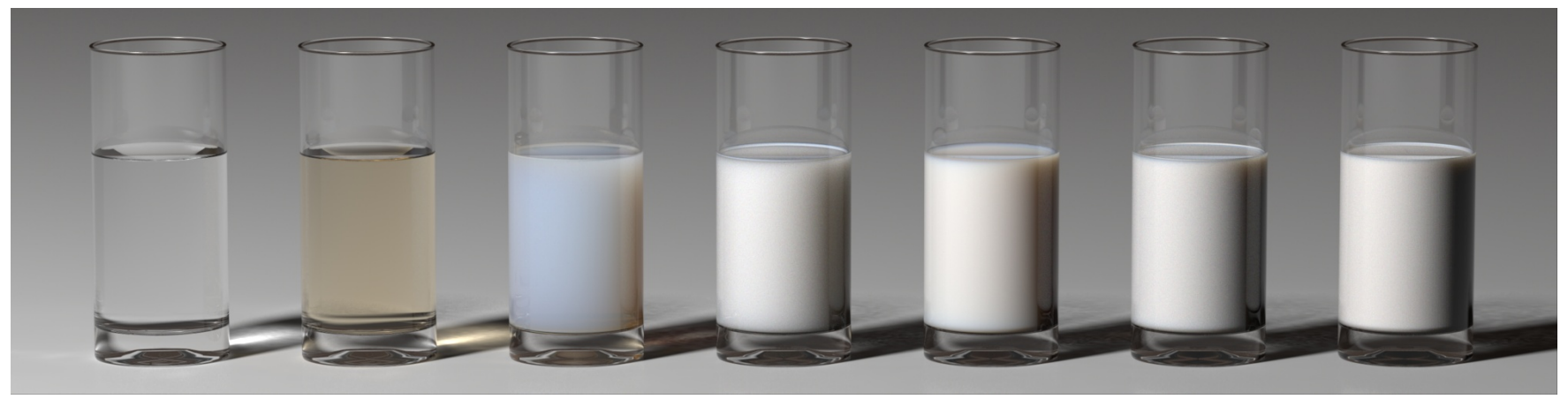

Figure 1: Rendered images of the components in milk as well as mixed concentrations. The optical properties of the components and the milk have been computed using the generalization of the Lorenz-Mie theory presented in this paper. From left to right the glasses contain: Water, water and vitamin B2, water and protein, water and fat, skimmed milk, regular milk, and whole milk.

\begin{abstract}
This paper introduces a theoretical model for computing the scattering properties of participating media and translucent materials. The model takes as input a description of the components of a medium and computes all the parameters necessary to render it. These parameters are the extinction and scattering coefficients, the phase function, and the index of refraction. Our theory is based on a robust generalization of the Lorenz-Mie theory. Previous models using Lorenz-Mie theory have been limited to non-absorbing media with spherical particles such as paints and clouds. Our generalized theory is capable of handling both absorbing host media and nonspherical particles, which significantly extends the classes of media and materials that can be modeled. We use the theory to compute optical properties for different types of ice and ocean water, and we derive a novel appearance model for milk parameterized by the fat and protein contents. Our results show that we are able to match measured scattering properties in cases where the classical LorenzMie theory breaks down, and we can compute properties for media that cannot be measured using existing techniques in computer graphics.
\end{abstract}

CR Categories: I.3.3 [Computer Graphics]: Picture/Image Generation; I.3.7 [Computer Graphics]: 3D Graphics and Realism

Keywords: Lorenz-Mie theory, participating media, optical properties, appearance modeling, realistic rendering.

(C)ACM, 2007. This is the author's version of the work. It is posted here by permission of ACM for your personal use. Not for redistribution. The definitive version was published in ACM Transactions on Graphics, Vol. 26, No. 3, Article 60, July 2007.

\section{Introduction}

Participating media and scattering materials are all around us. Examples include the atmosphere, ocean water, clouds, and translucent materials such as milk, ice, and human skin. In order to render these media, it is necessary to know the optical properties that describe how light interacts with the media. These scattering properties can be acquired by measurements, by manual adjustment to match the desired appearance, or by use of a theoretical model.

In this paper, we present a novel theoretical model capable of computing the scattering properties of a large class of natural media and materials. Our model is based on the LorenzMie theory [Lorenz 1890; Mie 1908], which is a complete solution to Maxwell's equation for scattering of electromagnetic waves by a homogeneous, spherical particle embedded in a nonabsorbing medium. This theory was introduced to graphics by Rushmeier [1995], and it has been used to compute the optical properties of pigmented materials [Callet 1996], for example paints and plastics in a transparent solvent, and for rendering of atmospheric phenomena [Jackèl and Walter 1997; Riley et al. 2004].

The classical Lorenz-Mie theory is limited to spherical particles in a non-absorbing host medium. This is one of the reasons why it has only been applied to pigmented materials and atmospheric phenomena such as clouds of round water droplets. For many other media and materials the host is absorbing. The refractive index of an absorbing host is a complex number. This complex index of refraction cannot be used directly with the classical theory since the standard evaluation techniques become unstable. We present a generalized version of the theory which includes robust evaluation formulas for the case where the host medium is absorbing. We show how the correct scattering properties are obtained when the refractive index of the host medium is complex. The computed scattering properties are spectral values for the scattering and absorption coefficients, the phase functions, and the index of refraction. We also present formulas for non-spherical particles. Our extensions are based in part on new developments in related fields as well as new contributions that make the theory useful in computer graphics.

Our results include a novel appearance model for milk capable of predicting the scattering properties based on the concentration of fat and protein in the milk (see Figure 1). This appearance model 
is in good agreement with previous measurements of milk. We also include examples of ice with non-spherical inclusions as well as various types of ocean water. In both these cases the host absorption is visually significant as it is the reason for the expected bluish appearance of the media. We believe the generalized Lorenz-Mie theory will provide a useful framework for computing the scattering properties of participating media and scattering materials. To encourage use of the theory, the source code of our implementation is published as a supplement to this paper.

\subsection{Related Work}

In recent years a number of practical methods have been developed for measuring the scattering properties of translucent materials and participating media. Jensen et al. [2001] developed a method based on diffusion theory and measured the absorption and reduced scattering coefficients of translucent materials, Tong et al. [2005] presented a method for measuring the properties of quasi-homogeneous translucent materials, Hawkins et al. [2005] developed a laser scanning system for estimating the albedo and phase function for time varying participating media such as smoke, and Narasimhan et al. [2006] presented a method based on dilution for measuring the scattering and absorption coefficients as well as the phase function. Our theory provides a number of advantages in comparison to methods based on measurements as it can (a) compute all properties at any desired wavelength, (b) predict the appearance of a material based on a mix of components without actually having the material, and (c) compute properties for materials (such as ice) that cannot be measured using existing techniques.

\section{Scattering in Participating Media}

The propagation of light in participating media is described by the radiative transfer equation [Chandrasekhar 1950]:

$$
(\vec{\omega} \cdot \nabla) L(\boldsymbol{x}, \vec{\omega})=-\sigma_{t} L(\boldsymbol{x}, \vec{\omega})+\sigma_{s} \int_{4 \pi} p\left(\vec{\omega}^{\prime}, \vec{\omega}\right) L\left(\boldsymbol{x}, \vec{\omega}^{\prime}\right) \mathrm{d} \omega^{\prime}
$$

where $L(\boldsymbol{x}, \vec{\omega})$ is the radiance at $\boldsymbol{x}$ in direction $\vec{\omega}$, and $\sigma_{t}, \sigma_{s}$, and $\sigma_{a}=\sigma_{t}-\sigma_{s}$ are the extinction, scattering, and absorption coefficients. The phase function $p$ specifies the normalized distribution of the scattered light. The radiative transfer equation provides a macroscopic view of scattering and absorption within a participating medium, and the only required parameters are $\sigma_{t}, \sigma_{s}$, and $p$.

Participating media are often composed of tiny particles that scatter and absorb light. It is the combined effect of these particles that provides the participating medium with its overall scattering properties. Lorenz-Mie theory makes it possible to compute the scattering properties of a single homogeneous, spherical particle embedded in a homogeneous medium. The scattering properties are computed using the scattering amplitude functions $S_{1}$ and $S_{2}$, which describe the scattering of an electromagnetic wave from a spherical particle [Lorenz 1890; Mie 1908]:

$$
\begin{aligned}
& S_{1}(\theta)=\sum_{n=1}^{\infty} \frac{2 n+1}{n(n+1)}\left(a_{n} \pi_{n}(\cos \theta)+b_{n} \tau_{n}(\cos \theta)\right) \\
& S_{2}(\theta)=\sum_{n=1}^{\infty} \frac{2 n+1}{n(n+1)}\left(b_{n} \pi_{n}(\cos \theta)+a_{n} \tau_{n}(\cos \theta)\right) .
\end{aligned}
$$

The functions $\pi_{n}$ and $\tau_{n}$ are related to the Legendre polynomials $P_{n}$ as follows:

$$
\begin{aligned}
\pi_{n}(\mu) & =\frac{d P_{n}(\mu)}{d \mu} \\
\tau_{n}(\mu) & =\mu \pi_{n}(\mu)-\left(1-\mu^{2}\right) \frac{d \pi_{n}(\mu)}{d \mu} .
\end{aligned}
$$

Their numeric evaluation can be found in standard references on Lorenz-Mie theory [Dave 1969; Bohren and Huffman 1983].

The coefficients $a_{n}$ and $b_{n}$ are complex numbers called the Lorenz-Mie coefficients and computation of these is fairly involved. Until recently the calculation of $a_{n}$ and $b_{n}$ has been carried out assuming that the host medium is non-absorbing [van de Hulst 1957,1981; Bohren and Huffman 1983; Callet 1996]. This assumption simplifies the computation because the refractive index of the host medium is then real-valued. This is due to the following fundamental relation between the absorption coefficient $\sigma_{a}$ of a substance and its complex index of refraction $\eta$ [Born and Wolf 1999]:

$$
\sigma_{a}=4 \pi \operatorname{Im}(\eta) / \lambda_{0}
$$

where $\lambda_{0}$ is the wavelength of light in vacuum and Im takes the imaginary part of a complex number.

\subsection{Particles Embedded in an Absorbing Medium}

The Lorenz-Mie coefficients $a_{n}$ and $b_{n}$ in Equations 2 and 3, are composed of the spherical Bessel functions $j_{n}(z)$ and $y_{n}(z)$ in the following way [Lorenz 1890]:

$$
\begin{aligned}
\psi_{n}(z) & =z j_{n}(z) \\
\zeta_{n}(z) & =z\left(j_{n}(z)-\boldsymbol{i} y_{n}(z)\right) \\
a_{n} & =\frac{\eta_{\text {med }} \psi_{n}^{\prime}(y) \psi_{n}(x)-\eta \psi_{n}(y) \psi_{n}^{\prime}(x)}{\eta_{\text {med }} \psi_{n}^{\prime}(y) \zeta_{n}(x)-\eta \psi_{n}(y) \zeta_{n}^{\prime}(x)} \\
b_{n} & =\frac{\eta \psi_{n}^{\prime}(y) \psi_{n}(x)-\eta_{\text {med }} \psi_{n}(y) \psi_{n}^{\prime}(x)}{\eta \psi_{n}^{\prime}(y) \zeta_{n}(x)-\eta_{\text {med }} \psi_{n}(y) \zeta_{n}^{\prime}(x)} .
\end{aligned}
$$

In these equations the primes ' denote derivative and $\eta_{\text {med }}$ and $\eta$ are the refractive indices of the host medium and the particle, respectively. The size parameters $x$ and $y$ are defined by:

$$
x=2 \pi r \eta_{\text {med }} / \lambda_{0} \quad, \quad y=2 \pi r \eta / \lambda_{0},
$$

where $r$ is the radius of the spherical particle considered.

When the host medium is non-absorbing, the arguments to $\zeta_{n}(x)$ and its derivative are real, and this makes it easy to obtain robust recurrence formulas for their evaluation [Dave 1969; Bohren and Huffman 1983]. In the case of an absorbing host medium, these formulas become unstable as they enter the exponential domain and run out of bounds. To avoid this ill-conditioning, the Lorenz-Mie coefficients can be rewritten in a form involving only ratios between the variations $\psi_{n}$ and $\zeta_{n}$ of the spherical Bessel functions [Kattawar and Plass 1967]

$$
\begin{aligned}
a_{n} & =\frac{\psi_{n}(x)}{\zeta_{n}(x)} \frac{\eta_{\mathrm{med}} A_{n}(y)-\eta A_{n}(x)}{\eta_{\mathrm{med}} A_{n}(y)-\eta B_{n}(x)} \\
b_{n} & =\frac{\psi_{n}(x)}{\zeta_{n}(x)} \frac{\eta A_{n}(y)-\eta_{\mathrm{med}} A_{n}(x)}{\eta A_{n}(y)-\eta_{\mathrm{med}} B_{n}(x)}
\end{aligned}
$$

Here $A_{n}(z)$ and $B_{n}(z)$ denote the logarithmic derivatives of $\psi_{n}(z)$ and $\zeta_{n}(z)$ respectively,

$$
A_{n}(z)=\frac{\psi_{n}^{\prime}(z)}{\psi_{n}(z)} \quad, \quad B_{n}(z)=\frac{\zeta_{n}^{\prime}(z)}{\zeta_{n}(z)} .
$$

The ratio $A_{n}$ is only numerically stable with downward recurrence. Therefore the following formula is employed for its evaluation [Kattawar and Plass 1967]

$$
A_{n}(z)=\frac{n+1}{z}-\left(\frac{n+1}{z}+A_{n+1}(z)\right)^{-1} .
$$

This formula is also valid for the ratio $B_{n}$, but unfortunately it is unstable for both upward and downward recurrence [Cachorro and 
Salcedo 1991]. Instead, we use a different formula for $B_{n}$ which has been developed by Mackowski et al. [1990] in the field of multilayered particles embedded in a non-absorbing medium. Mackowski et al. developed the formula to compute scattering within a multilayered particle, but it can also be applied in our case, and it is numerically stable with upward recurrence for any complex argument [Mackowski et al. 1990]:

$$
\begin{aligned}
B_{n}(z)= & A_{n}(z)+\frac{\boldsymbol{i}}{\psi_{n}(z) \zeta_{n}(z)} \\
\psi_{n}(z) \zeta_{n}(z)= & \psi_{n-1}(z) \zeta_{n-1}(z) \\
& \times\left(\frac{n}{z}-A_{n-1}(z)\right)\left(\frac{n}{z}-B_{n-1}(z)\right) .
\end{aligned}
$$

It remains to give a recurrence relation for the ratio $\psi_{n}(z) / \zeta_{n}(z)$ in Equations 12 and 13. Recent developments in the context of multilayered particles, provide a recurrence relation that works well for small $\operatorname{Im}(z)$ [Wu and Wang 1991; Yang 2003]:

$$
\frac{\psi_{n}(z)}{\zeta_{n}(z)}=\frac{\psi_{n-1}(z)}{\zeta_{n-1}(z)} \frac{B_{n}(z)+n / z}{A_{n}(z)+n / z}
$$

The restriction to small $\operatorname{Im}(z)$ is not a problem, as a $\operatorname{larger} \operatorname{Im}(z)$ means that the host medium is highly absorbing, and in this case we would not be able to make out the effect of particle scattering.

The amplitude functions $(2,3)$ are defined by an infinite sum, and in order to get a decent approximation, we must find an appropriate number of terms $M$ to sum. This is also necessary for initialization of the downward recurrence (15) computing $A_{n}(x)$ and $A_{n}(y)$. A formula determining $M$, which has both an empirical [Wiscombe 1980; Mackowski et al. 1990] and a theoretical [Cachorro and Salcedo 1991] justification, is

$$
M=\left\lceil|x|+p|x|^{1 / 3}+1\right\rceil,
$$

where $p=4.3$ gives a maximum error of $10^{-8}$. It is possible to calculate an approximate initial value for the downward recurrence (15), but, as explained by Dave [1969], the recurrence is not sensitive to the initial value, and therefore we can arbitrarily choose $A_{M}(z)=0$.

Once $A_{0}(z), \ldots, A_{M}(z)$ are computed for both $z=x$ and $z=$ $y$, we can compute $B_{n}(x), \psi_{n}(x) / \zeta_{n}(x), a_{n}$ and $b_{n}$ step by step. Note, that there is no need to store $B_{n}(x)$ and $\psi_{n}(x) / \zeta_{n}(x)$ since they are computed using upward recurrences. These recurrences should be initialized by

$$
\begin{aligned}
B_{0}(z) & =\boldsymbol{i} \\
\psi_{0}(z) \zeta_{0}(z) & =\frac{1}{2}\left(1-e^{i 2 z}\right) \\
\psi_{0}(z) / \zeta_{0}(z) & =\frac{1}{2}\left(1-e^{-i 2 z}\right) .
\end{aligned}
$$

Recall that there is a direct relationship between wavelength $\lambda_{0}$ and the size parameters $x$ and $y$. This tells us that the LorenzMie coefficients are spectrally dependent and should preferably be sampled at different wavelengths. They are also dependent on the particle radius $r$ and are valid for spherical particles of arbitrary size as long as they do not exhibit diffuse reflection (which is only possible if the particle size greatly exceeds the wavelength and even so, the surface of the particle might still be smooth) [van de Hulst 1957,1981]. Furthermore the equations provided in this section reveal that the complex refractive index of each particle inclusion, as well as that of the host medium, are needed as input parameters for the computations.

Having a robust way to compute the Lorenz-Mie coefficients we can evaluate the scattering amplitudes using Equations 2 and 3. Given the scattering amplitudes we can find the extinction and scattering cross sections as well as the phase function of the particle.
These are all well defined quantities for particles in a non-absorbing medium. In an absorbing medium, there is, however, no unambiguous way to determine the part of the extinction which is due to scattering by the particle, because the medium is part of the extinction process [Bohren and Gilra 1979; Videen and Sun 2003]. Often the resulting formulas depend on the size of an arbitrary sphere used only as a mathematical tool for integration. Such expressions are not physically meaningful and will not be considered here. Both near-field and far-field effects are discussed in the literature. We will only consider far-field approximations as they correspond to a distant observer. Near-field effects are not generally relevant in graphics as we mostly want to estimate the bulk optical properties of a participating medium.

Due to the problems discussed above, the extinction cross section, $C_{t}$, is the only well defined observable quantity [Bohren and Gilra 1979]. It is computed using an optical theorem first presented by van de Hulst $[1949 ; 1957,1981]$. The original theorem by van de Hulst is valid for particles of arbitrary shape and size, but it only applies to a non-absorbing host medium. To account for an absorbing host, we use a slightly modified equation presented by Bohren and Gilra [1979]:

$$
C_{t}=4 \pi \operatorname{Re}\left(\frac{S(0)}{k^{2}}\right),
$$

where $S(0)=S_{1}(0)=S_{2}(0)$ is the amplitude in the forward direction of the scattered wave, $k=2 \pi \eta_{\text {med }} / \lambda_{0}$ is the wave number, and Re takes the real part of a complex number. Inserting the expression for $S(0)$ in this optical theorem (21) we obtain

$$
C_{t}=\frac{\lambda_{0}^{2}}{2 \pi} \sum_{n=1}^{\infty}(2 n+1) \operatorname{Re}\left(\frac{a_{n}+b_{n}}{\eta_{\text {med }}^{2}}\right) .
$$

The scattering cross section $C_{s}$ cannot be found unambiguously, but we have to approximate it to evaluate the radiative transfer equation (1) in a rendering. We use a far-field approximation which has been reported to be consistent with measured data [Randrianalisoa et al. 2006; Yin and Pilon 2006]. The chosen formula is identical to the scattering cross section for transparent media except for two correction terms; an exponential term and a geometrical term $\gamma$. The formula is

$$
C_{s}=\frac{\lambda_{0}^{2} e^{-4 \pi r \operatorname{Im}\left(\eta_{\mathrm{med}}\right) / \lambda_{0}}}{2 \pi \gamma\left|\eta_{\mathrm{med}}\right|^{2}} \sum_{n=1}^{\infty}(2 n+1)\left(\left|a_{n}\right|^{2}+\left|b_{n}\right|^{2}\right)
$$

where $r$ in the exponential term is the uncertain part of the equation because it ought to be the distance to where the scattered wave is observed. This distance is unknown, and consequently it has been projected to the particle surface, such that $r$ denotes the particle radius.

The geometrical term $\gamma$ accounts for the fact that the incident wave changes over the surface of the particle as a consequence of the absorbing host medium. It is defined by [Mundy et al. 1974]

$$
\gamma=\frac{2\left(1+(\alpha-1) e^{\alpha}\right)}{\alpha^{2}}
$$

where $\alpha=4 \pi r \operatorname{Im}\left(\eta_{\text {med }}\right) / \lambda_{0}$ and $\gamma \rightarrow 1$ for $\alpha \rightarrow 0$.

The precision of the far field approximation $(23,24)$ has recently been reviewed [Fu and Sun 2006] and compared to experimental data [Randrianalisoa et al. 2006; Yin and Pilon 2006]. The conclusion is that it (as expected) does not give entirely accurate results, but it does give physically plausible results. It is also concluded that significant errors can result if the absorption of the host medium is ignored (this is especially true when the size parameter $x$ is large). 
The phase function and the asymmetry parameter are the same in transparent and absorbing media [Yang et al. 2002]. The phase function for unpolarized light is [van de Hulst 1957,1981]

$$
p(\theta)=\frac{\left|S_{1}(\theta)\right|^{2}+\left|S_{2}(\theta)\right|^{2}}{4 \pi \sum_{n=1}^{\infty}(2 n+1)\left(\left|a_{n}\right|^{2}+\left|b_{n}\right|^{2}\right)} .
$$

The asymmetry parameter (which is defined by the integral over the cosine weighted phase function in all directions) is [van de Hulst 1957,1981]:

$g=\frac{\sum_{n=1}^{\infty}\left\{\frac{n(n+2)}{n+1} \operatorname{Re}\left(a_{n} a_{n+1}^{*}+b_{n} b_{n+1}^{*}\right)+\frac{2 n+1}{n(n+1)} \operatorname{Re}\left(a_{n} b_{n}^{*}\right)\right\}}{\frac{1}{2} \sum_{n=1}^{\infty}(2 n+1)\left(\left|a_{n}\right|^{2}+\left|b_{n}\right|^{2}\right)}$,

where the asterisks $*$ denote the complex conjugate.

\subsection{Bulk Optical Properties}

To determine the macroscopic (or phenomenological) optical properties of a bulk medium in which many small particles are immersed, it is common to assume that the scattering by one particle can be considered independent of the scattering by another. This is a good approximation as long as the medium is not extremely dense (meaning that the distance between the particles should be considerably larger than $\lambda_{0}$ [van de Hulst 1957,1981]). If this is not the case, the radiative transfer equation (1) cannot be used. Supposing that particles scatter light independently in the medium, the bulk extinction and scattering coefficients are given by [van de Hulst 1957,1981]

$$
\sigma_{j}\left(\lambda_{0}\right)=\int_{0}^{\infty} C_{j}\left(r, \lambda_{0}\right) N(r) \mathrm{d} r
$$

where $r$ is the radius of a particle, $N(r)$ is the particle number density distribution, and $j$ is either $t$ referring to extinction or $s$ referring to scattering. Of course the integral disappears if the particles are all the same size. But in most natural materials a single particle radius cannot predict the optical properties correctly. The integral will, however, always be zero outside some limited interval $\left[r_{\min }, r_{\max }\right]$ of particle sizes.

Particle size distribution is the common term for distributions by which the number density can be derived. Particle sizes often follow a log-normal distribution which is described by a mean particle size $\mu$ and a coefficient of variation $c_{v}=\sigma / \mu$, where $\sigma$ is the standard deviation. For completeness we include equations in Appendix A for the log-normal distribution and the power law distribution which is common for larger particles.

In the following, let $A$ denote the set of homogeneous substances appearing as particles in the host medium. The number density $N(r) \mathrm{d} r$ specifies the number of particles per unit volume with radii in the interval $\mathrm{d} r$, and we can find the volume fraction occupied by a particle inclusion consisting of spherical particles by

$$
v=\frac{4 \pi}{3} \int_{r_{\min }}^{r_{\max }} r^{3} N(r) \mathrm{d} r .
$$

This is important since, in almost any case, either the number density distribution $N(r)$ or the volume frequency $r^{3} N(r)$ is a measurable particle size distribution. If we have an empirical function or tabulated data describing either of the size distributions, we can evaluate the integral (28) and find the volume fraction $v_{\text {original }}$ of the particles in the sample from which the size distribution was originally estimated. This may not be the volume fraction we desire in our medium. Suppose the volume fraction $v_{i}, i \in A$, is desired rather than $v_{\text {original }}$. Then the measured number density should be scaled by $v_{i} / v_{\text {original }}$.
Because we assume that particles scatter light independently, interference between scattered waves can be ignored for the bulk medium. This means that wave intensities can be summed to obtain, for example, a combined scattered wave [van de Hulst 1957,1981]. This is the assumption behind the integral over particle radii (27) which finds coefficients using cross sections. The same assumption is used when we go from scattering and extinction coefficients, $\sigma_{s, i}$ and $\sigma_{t, i}$, for every individual particle inclusion $i \in A$, to combined scattering and extinction coefficients for the bulk medium. For the scattering coefficient the summation is straight forward:

$$
\sigma_{s}=\sum_{i \in A} \sigma_{s, i}
$$

Note that volume fractions are not included in this formula, because they are a part of the number density distributions.

In a transparent medium, the extinction coefficient is defined by an equivalent sum, but in an absorbing medium an important correction must be made. Since the host medium is a part of the extinction process, a non-absorbing particle will reduce the extinction of the bulk medium. This means that the extinction cross sections can be negative [Bohren and Gilra 1979]. The extinction cross section resulting from the Lorenz-Mie theory is, in other words, relative to the absorption of the host medium and the necessary correction is to include the host medium absorption in the sum. For this purpose, we compute the bulk extinction coefficient for particles in an absorbing medium by

$$
\sigma_{t}=\sigma_{a, \mathrm{med}}+\sum_{i \in A} \sigma_{t, i}
$$

and the bulk absorption coefficient is given by the simple relation $\sigma_{a}=\sigma_{t}-\sigma_{s}$. These bulk coefficients are never negative.

The asymmetry parameter (26) and phase function (25) are normalized properties related to the amount of scattering by every particle. Say we denote the asymmetry parameter of a single particle $g_{p}(r)$ and the corresponding phase function $p_{p}(r)$, where $r$ is the particle radius. The ensemble asymmetry parameter $g_{i}$ and phase function $p_{i}$ including all the different sizes of a particular type $i \in A$, are then found by a weighted average, where the weights are the associated scattering cross sections. In other words, for a particle inclusion $i \in A$ :

$g_{i}=\frac{1}{\sigma_{s}} \int_{r_{\min }}^{r_{\max }} C_{s}(r) g_{p}(r) \mathrm{d} r \quad, \quad p_{i}(\theta)=\frac{1}{\sigma_{s}} \int_{r_{\min }}^{r_{\max }} C_{s}(r) p_{p}(\theta, r) \mathrm{d} r$.

Once the scattering properties have been determined for each individual particle inclusion, the bulk properties are computed using a weighted average [Grenfell 1983; Light et al. 2004]:

$$
g=\frac{\sum_{i \in A} \sigma_{s, i} g_{i}}{\sigma_{s}} \quad, \quad p(\theta)=\frac{\sum_{i \in A} \sigma_{s, i} p_{i}(\theta)}{\sigma_{s}} .
$$

Considering the number of Lorenz-Mie expressions required to approximate the true Lorenz-Mie phase function $p(\theta)$, it is more practical to either tabulate the phase function or use a mean number density for each particle inclusion in order to avoid the integral (31). It is also possible to use the bulk asymmetry parameter $g$ in Equation 32 with one of the standard phase functions, e.g. the HenyeyGreenstein phase function [Henyey and Greenstein 1940], or a multilobed phase function where the Henyey-Greenstein function replaces $p_{i}(\theta)$ in Equation 32.

To compute the refractive index of the bulk medium, we follow van de Hulst's [1957,1981] derivation of a formula for the effective index of refraction, but remove the assumptions of non-absorbing 
media and particles of only one radius. This gives the following approximate relation for the real part of the bulk refractive index:

$$
\begin{aligned}
& \operatorname{Re}\left(\eta_{\text {bulk }}\left(\lambda_{0}\right)\right)=\operatorname{Re}\left(\eta_{\text {med }}\left(\lambda_{0}\right)\right) \\
& \quad+\lambda_{0} \sum_{i \in A} \int_{0}^{\infty} \operatorname{Im}\left(\frac{S_{i, r, \lambda_{0}}(0)}{k^{2}}\right) N_{i}(r) \mathrm{d} r .
\end{aligned}
$$

The imaginary part of the effective index of refraction is not the correct imaginary part for the bulk medium, but rather a term related to the total extinction of the medium. The correct imaginary part is found by its relation to the bulk absorption coefficient (6).

\subsection{Non-Spherical Particles}

Lorenz-Mie theory is for spherical particles, but particles are not always spherical. If non-spherical particles are encountered, it has been found by Grenfell and Warren [1999] that volume-toarea equivalent spheres give a good approximation of how the nonspherical particles scatter light. To represent a particle of volume $V$ and surface area $A$ by a collection of spheres, the radius of the equivalent spheres is [Grenfell and Warren 1999]:

$$
r_{\mathrm{eq}}=3 \frac{V}{A} .
$$

Since the number of equivalent spheres is not equal to the number of non-spherical particles, the number density must be adjusted accordingly [Grenfell and Warren 1999]:

$$
\frac{N_{\mathrm{eq}}}{N}=\frac{3 V}{4 \pi r_{\mathrm{eq}}^{3}} \text {. }
$$

The equivalent radius $r_{\text {eq }}$ and the equivalent number density $N_{\text {eq }}$ are then used for computation of scattering and extinction coefficients (27) with Lorenz-Mie theory for computation of the corresponding cross sections.

As opposed to equal-volume and equal-area spheres, the volumeto-area equivalent spheres have proven to be quite exact. They have been tested for cylinders [Grenfell and Warren 1999], hexagonal columns and plates [Neshyba et al. 2003], and hollow columns and plates [Grenfell et al. 2005]. In most cases the error is less than 5\%. At least this is true for scattering and extinction coefficients. The approximation is, as could be expected, less accurate with respect to the phase function.

In Appendix B we have derived volume, surface area, and equivalent sphere radius for ellipsoids and cylinders. These can be used directly with Equations 35, 27, and 28.

\section{Rendering Using Similarity Theory}

The scattering properties computed using Lorenz-Mie theory can be used in most standard rendering systems capable of rendering participating media. Many natural materials are highly scattering as well as highly forward scattering, and these types of optical properties are particularly challenging for existing rendering algorithms. This includes the diffusion-based methods [Jensen et al. 2001] that cannot handle highly forward scattering materials or materials with significant absorption. Therefore we use Monte Carlo ray tracing for all our results. Monte Carlo ray tracing is very costly, but it ensures that the final renderings show the effect of the optical properties without other types of error. For some of the materials the number of scattering events that an average photon experiences is very high - for milk it is several thousand events. To reduce the computational requirements in these cases we make a minor modification to the Monte Carlo ray tracing algorithm using similarity theory. Similarity theory makes it possible to convert a medium with anisotropic scattering into a medium with isotropic scattering and still obtain the same overall appearance [Wyman et al. 1989].
This assumption holds true for light that has penetrated some distance into the medium. To take advantage of this property, we modify the Monte Carlo ray tracing such that it switches to a medium with isotropic scattering and uses a reduced scattering coefficient $\sigma_{s}^{\prime}=(1-g) \sigma_{s}$ when the path is longer than 10 mean free paths $\left(10 / \sigma_{t}\right)$. This reduces the number of scattering events and thereby the rendering time for highly forward scattering materials such as milk and ice by a factor 10-20. The error introduced by using similarity theory after 10 mean free paths is insignificant and has no influence on the rendered images.

\section{Examples}

In this section we demonstrate the generalized Lorenz-Mie theory on a number of different media. We have computed full spectral properties (sampled at every $25 \mathrm{~nm}$ ) for all the materials, and in all cases the time to compute these profiles was a few seconds. For rendering we computed RGB representations of the optical properties by weighted averages using the RGB functions for a standard human observer [Stockman and Sharpe 2000]. Alternatively, we could have used spectral rendering and tone-mapped the result to RGB. We chose an RGB representation in order to compare with existing measurements in graphics.

To compute the optical properties, we need complex indices of refraction for the homogeneous substances involved and size distributions for the particles. Indices of refraction (including the imaginary part) are often available in chemical software packages (e.g. PhotochemCAD [Du et al. 1998]), physics handbooks [Gray 1972; Palik 1985; Lide 2006], and online repositories (e.g. http://www.luxpop.com/). Particle size distributions can often be found in optics or chemistry literature, or some mean particle size can be estimated. Once the input parameters have been obtained for one substance, they may be used in many different contexts (cf. how minerals and algae are used for both water and ice below).

\subsection{Milk}

The optically important elements in milk include vitamin B2, fat globules and casein micelles (protein) [Crofcheck et al. 2002]. To model the concentration of fat and protein we use wt.-\% (g per $100 \mathrm{~g}$ milk), since this value is used on contents declarations on the side of milk cartons. In the remainder of this section we let $w_{f}$ and $w_{p}$ denote the wt.- $\%$ of fat and protein respectively. To translate wt. $-\%$ into volume fractions, we use the density of milk fat $\rho_{\text {fat }}=$ $0.915 \mathrm{~g} / \mathrm{mL}$, the density of milk protein $\rho_{\text {protein }}=1.11 \mathrm{~g} / \mathrm{mL}$, and the density of milk $\rho_{\text {milk }}=1.03 \mathrm{~g} / \mathrm{mL}$ [Walstra and Jenness 1984]. Casein micelles make up about $76 \%$ of the protein volume fraction [Fox and McSweeney 1998]. This means that

$$
v_{f}=\frac{w_{f} / \rho_{\mathrm{fat}}}{100 \mathrm{~g} / \rho_{\mathrm{milk}}} \quad, \quad v_{p}=0.76 \frac{w_{p} / \rho_{\text {protein }}}{100 \mathrm{~g} / \rho_{\text {milk }}} .
$$

The host medium is water in which many different components are dissolved. The component exhibiting the most significant absorption in the visible range is vitamin B2 (riboflavin). Spectral data for the absorption of vitamin B2 can be found in the PhotochemCAD application ${ }^{1}$ [Du et al. 1998], and in Appendix C we have listed the resulting imaginary part of the milk host medium. Note, that there is significant absorption for shorter wavelengths.

The Complex refractive index for milk fat has been reported by Michalski et al. [2001]. The real part is approximately $\operatorname{Re}\left(\eta_{\text {fat }}\right)=$ 1.46, the imaginary part is included in Appendix C. Casein micelles only scatter light and the refractive index is $\eta_{\text {casein }}=1.503$ in the visible range [Attaie and Richtert 2000].

The volume frequency of the fat globules follows a log-normal distribution [Walstra 1975] (see Appendix A). The mean of the

\footnotetext{
${ }^{1}$ See http://omlc.ogi.edu/spectra/PhotochemCAD/abs_html/riboflavin.html
} 
volume-to-surface area equivalent sphere radii $r_{\mathrm{vs}, \text { fat }}$ of the fat globules change depending on the volume fraction of the globules in the milk. By a least-squares, two-piece fit to measured data reported by Olson et al. [2004], we have found a functional expression describing this relationship:

$$
r_{43, \mathrm{fat}}=\left\{\begin{array}{cl}
-0.2528 w_{f}^{2}+1.419 w_{f} & \text { for } w_{f}<2.0 \\
1.456 w_{f}^{0.36} & \text { otherwise }
\end{array},\right.
$$

where $r_{43 \text {,fat }}$ is measured in $\mu \mathrm{m}$. The relationship between $r_{43, \text { fat }}$ and $r_{\mathrm{vs}, \text { fat }}$ is [Walstra 1975]

$$
r_{\mathrm{vs}, \mathrm{fat}}=r_{43, \mathrm{fat}} /\left(c_{v, \mathrm{fat}}^{2}+1\right) .
$$

The radius $r_{43 \text {,fat }}$ is used since it can be estimated with good accuracy [Walstra 1975]. The coefficient of variation $c_{v}$,fat is usually between 0.4 and 1.2 in normal milk. We use $c_{v}$,fat $=0.6$ in our computations. Reasonable limits for the range of fat globule radii are $r_{\text {min,fat }}=0.005 \mu \mathrm{m}$ and $r_{\max \text {,fat }}=10 \mu \mathrm{m}$.

For casein micelles Schmidt et al. [1973] found that $r_{\mathrm{vs}, \mathrm{cas}}=$ $43 \mathrm{~nm}$ and showed that a log-normal distribution (Appendix A) of $r /\left(r_{\max , \text { cas }}-r\right)$ fits the volume frequency distribution well. The limits for the casein micelle radii are $r_{\text {min,cas }}=0$ and $r_{\text {max,cas }}=$ $150 \mathrm{~nm}$.

All necessary input is now available such that we can compute the optical properties as follows:

1. Number density of fat globules and casein micelles are determined such that they constitute the desired volume fractions (Equations 28,49,50, and 50).

2. For every wavelength sampled and every particle radius considered, we evaluate the Lorenz-Mie coefficients $a_{n}$ and $b_{n}$ (Equations 19,15,20,16,17,18,12, and 13), and we compute the extinction cross section $C_{t}$, scattering cross section $C_{s}$, and asymmetry parameter $g_{p}$ (Equations 22,23,24, and 26).

3. Scattering coefficient $\sigma_{s, i}$, extinction coefficient $\sigma_{t, i}$, and combined asymmetry parameter $g_{i}$ are determined for each particle inclusion $i \in\{$ fat, casein $\}$ (Equations 27, and 31).

4. Finally bulk extinction coefficient $\sigma_{t}$, bulk scattering coefficients $\sigma_{s}$, bulk absorption coefficient $\sigma_{a}$, bulk asymmetry parameter $g$, and effective refractive index are computed (Equations 29,30,32, and 33).

This procedure can be used for arbitrary volume fractions of casein micelles and fat globules, and it is the same for other participating media if we swap fat globules and casein micelles for a different set of particles.

Figure 1 shows several rendered glasses containing water, vita$\min \mathrm{B} 2$, protein and fat in various combinations. The optical properties for all glasses containing protein or fat, have been computed using the theory we present. The resulting images of milk capture important visual properties such as the red shadow in skimmed milk and the increasingly white appearance as the percentage of fat is increased.

\section{An Appearance Model for Milk}

Being able to compute the scattering properties of milk, we can construct an appearance model parameterized by the amount of fat and protein in the milk. These two parameters can be adjusted to model most types of milk. We have obtained the following RGB vector functions by least squares fits to the computed optical properties:

$$
\sigma_{a}=\left[\begin{array}{l}
1.381-0.008600 w_{p}+1.209 w_{f} \\
2.201-0.01459 w_{p}+1.982 w_{f} \\
10.13-0.07048 w_{p}+4.170 w_{f}
\end{array}\right]
$$

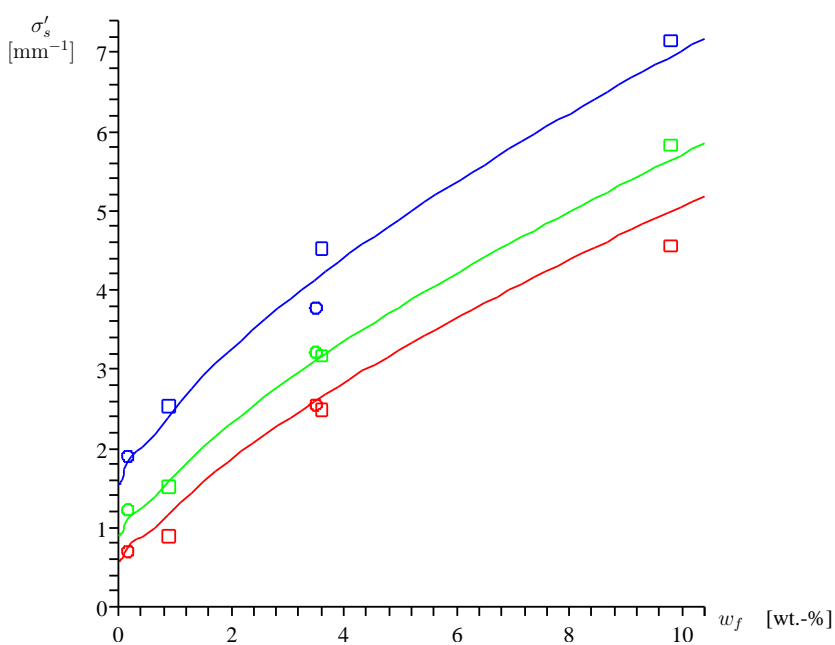

Figure 2: Reduced scattering coefficients $\sigma_{s}^{\prime}=(1-g) \sigma_{s}$ measured by Jensen et al. [2001] (circles) and Narasimhan et al. [2006] (squares) and plotted against our appearance model for milk as a function of fat content $w_{f}$. Protein content is constant at $3 w t .-\%$.

$$
\begin{aligned}
\sigma_{s}= & {\left[\begin{array}{l}
213.5 w_{p}+15631 w_{f}^{1.24} e^{h_{1}\left(\ln \left(w_{f}\right)\right)_{R}} \\
338.3 w_{p}+18349 w_{f}^{1.15} e^{h_{1}\left(\ln \left(w_{f}\right)\right)_{G}} \\
614.0 w_{p}+22585 w_{f}^{1.01} e^{h_{1}\left(\ln \left(w_{f}\right)\right)_{B}}
\end{array}\right] } \\
g= & {\left[\begin{array}{l}
\left(18.63 w_{p}+\left(\sigma_{s R}-213.5 w_{p}\right) \tilde{g}\left(w_{f}\right)_{R}\right) / \sigma_{s R} \\
\left(37.79 w_{p}+\left(\sigma_{s G}-338.3 w_{p}\right) \tilde{g}\left(w_{f}\right)_{G}\right) / \sigma_{s G} \\
\left(96.69 w_{p}+\left(\sigma_{s B}-614.0 w_{p}\right) \tilde{g}\left(w_{f}\right)_{B}\right) / \sigma_{s B}
\end{array}\right] . }
\end{aligned}
$$

A two-piece fit is needed for the asymmetry parameter, and consequently the $\tilde{g}$ function in Equation 41 is given by

$$
\begin{aligned}
\tilde{g}\left(0<w_{f}<0.7\right)= & {\left[\begin{array}{l}
0.9523 w_{f}^{-0.0120} e^{h_{2}\left(\ln \left(1 / w_{f}\right)\right)_{R}} \\
0.9539 w_{f}^{-0.00783} e^{h_{2}\left(\ln \left(1 / w_{f}\right)\right)_{G}} \\
0.9554 w_{f}^{-0.000161} e^{h_{2}\left(\ln \left(1 / w_{f}\right)\right)_{B}}
\end{array}\right] } \\
\tilde{g}\left(w_{f} \geq 0.7\right)= & {\left[\begin{array}{l}
0.9576 w_{f}^{0.00911} e^{h_{3}\left(\ln \left(w_{f}\right)\right)_{R}} \\
0.9585 w_{f}^{0.00783} e^{h_{3}\left(\ln \left(w_{f}\right)\right)_{G}} \\
0.9577 w_{f}^{0.00531} e^{h_{3}\left(\ln \left(w_{f}\right)\right)_{B}}
\end{array}\right] . }
\end{aligned}
$$

If $w_{f}=0$, replace $\tilde{g}$ by $\mathbf{0}$ and $\ln \left(w_{f}\right)$ by 0 . Finally the functions $h_{1}, h_{2}$, and $h_{3}$ are the following RGB vector polynomials

$$
\begin{aligned}
& h_{1}(x)=\left[\begin{array}{l}
-0.00129 x^{4}+0.0305 x^{3}-0.219 x^{2} \\
-0.00149 x^{4}+0.0327 x^{3}-0.213 x^{2} \\
-0.00206 x^{4}+0.0373 x^{3}-0.202 x^{2}
\end{array}\right] \\
& h_{2}(x)=\left[\begin{array}{l}
-0.0386 x^{3}-0.00543 x^{2} \\
-0.0368 x^{3}+0.00266 x^{2} \\
-0.0334 x^{3}+0.0111 x^{2}
\end{array}\right] \\
& h_{3}(x)=\left[\begin{array}{l}
0.000281 x^{3}-0.00366 x^{2} \\
0.000379 x^{3}-0.00401 x^{2} \\
0.000509 x^{3}-0.00429 x^{2}
\end{array}\right]
\end{aligned}
$$

The absorption and scattering coefficients in this appearance model have the unit $\mathrm{m}^{-1}$. For every band of the three optical properties $(39,40,41)$ the maximum error is $10.2 \%$. This error band only excludes input parameters where $w_{f}<0.05$ wt.- $\%$. The maximum error occurs in the blue band of the absorption coefficient. For the majority of the possible input parameters, the error rarely exceeds $2 \%$ in all bands of all properties.

In Figure 2, we have plotted measurements by Jensen et al. [2001] and by Narasimhan et al. [2006] against the curves given by our milk model when protein content is $w_{p}=3 \mathrm{wt}$. $-\%$. Since the contents of the milk used for the measurements are not known, we have placed the measurements where they best fit the curves. The fat content estimated for the measurements by Jensen et al. [2001] 


\begin{tabular}{lll}
\hline Region & $v_{\text {algal }}$ & $v_{\text {mineral }}$ \\
\hline Atlantic & $1.880 \cdot 10^{-7}$ & $2.477 \cdot 10^{-10}$ \\
Baltic & $1.904 \cdot 10^{-6}$ & $5.429 \cdot 10^{-7}$ \\
Channel & $4.999 \cdot 10^{-7}$ & $2.300 \cdot 10^{-7}$ \\
Mediterranean & $3.878 \cdot 10^{-7}$ & $3.075 \cdot 10^{-7}$ \\
North Sea & $2.171 \cdot 10^{-6}$ & $2.077 \cdot 10^{-6}$ \\
\hline
\end{tabular}

Table 1: Volume fraction of algae and minerals contained in coastal and oceanic waters around Europe. Data provided by Babin et al. [2003a] have been translated into the volume fractions given here.

could very well be the fat content of the original milk. For the measurements by Narasimhan et al. [2006] we had to overestimate the fat content (except for the lowfat case). The reason for this discrepancy is very likely due to problems caused by the dilution used for the measurements. If we increase the mean particle size and coefficient of variation in the fat globule size distribution, we are able to obtain results almost identical to the measurements by Narasimhan et al. [2006] without over-estimation of the fat contents. An increase of these two parameters corresponds exactly to the effect of milk dilution [Walstra 1975]. Dilution also explains the unexpected behavior of the asymmetry parameters measured by Narasimhan et al. [2006]. As compared to our computations, they seem to start out too high and decrease too quickly with increasing fat content.

Because milk is highly scattering, its absorption coefficient is very difficult to measure. Measured absorption coefficients are, however, still of the same order of magnitude as the predicted ones and they all exhibit the behavior $\sigma_{a, R}<\sigma_{a, G}<\sigma_{a, B}$. If we had ignored the absorption in the host medium in the Lorenz-Mie calculations, the absorption coefficient would only depend on fat content and would under-estimate the absorption in the blue band by more than a factor 48 for skimmed milk, while the smallest error is in the red band where it is $-96 \%$.

\subsection{Natural Waters}

The key ingredients in natural water are suspended algae and minerals. Deep water would appear black (except for the surface reflection) in the absence of scattering particles. For natural waters we use the following particle properties reported by Babin et al. [2003b; 2003a]:

\begin{tabular}{lll}
\hline Input & Mineral & Algae \\
\hline $\operatorname{Re}(\eta)$ & 1.58 & 1.41 \\
$\operatorname{Im}(\eta)$ & Appendix C & Appendix C \\
$r$ & $0.01-100 \mu \mathrm{m}$ & $0.225-100 \mu \mathrm{m}$ \\
$N(r)$ & $\frac{v_{\text {mineral }}}{10.440}(2 r)^{-3.4}$ & $\frac{v_{\text {algal }}}{4.9735}(2 r)^{-3.6}$ \\
\hline
\end{tabular}

For the host medium we use $\operatorname{Re}\left(\eta_{\text {brine }}\right)=1.34$ and the imaginary part for the refractive index for brine given in Appendix C.

Common volume fractions for different seas around Europe are given in Table 1, and Figure 3 shows rendered images of four different marine areas. The areas are all illuminated by the same atmospheric lighting model and the color difference in the images are only due to the optical properties of the water. Note, how increasing the amount of minerals gives the water a lighter blue color. The blue color is due to absorption in the water (host) medium. Increasing the amount of algae makes the water more green, which can be seen in the rendering of the North Sea.

\subsection{Ice}

Ice in itself is a pure substance, but in reality we hardly ever see it in its pure form. Most ice contains air particles and sea ice also contains brine pockets. In addition, minerals and algae may be present

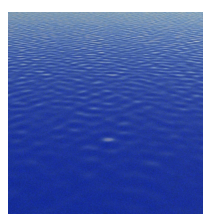

Atlantic

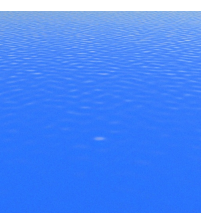

Mediterranean

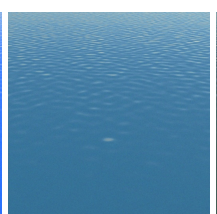

Baltic

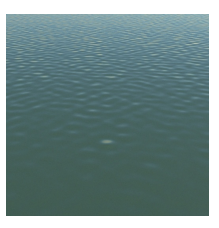

North Sea
Figure 3: Rendered images of different types of coastal and oceanic waters of increasing mineral and algal content.

in the ice. The latter two particle types were addressed in the previous section. For brine and air we use the following properties:

\begin{tabular}{lll}
\hline Input & Brine & Air \\
\hline $\operatorname{Re}(\eta)$ & 1.34 & 1.00 \\
$\operatorname{Im}(\eta)$ & Appendix C & 0.0 \\
$r$ & $0-14.6 \mathrm{~mm}$ & $0.1-2.0 \mathrm{~mm}$ \\
$N(r)$ & see below & $\frac{v_{\text {mineral }}}{10.278} r^{-1.24}$ \\
\hline
\end{tabular}

Spectral data for the complex refractive index of ice have been compiled by Warren [1984]. In the visible range, The real part is almost constant at $\operatorname{Re}\left(\eta_{\text {ice }}\right)=1.31$. The imaginary part can be found in Appendix C.

When water freezes in the sea, brine is trapped between vertical platelets of pure ice [Grenfell 1983]. If the freeze continues (below $-2{ }^{\circ} \mathrm{C}$ ) the initial platelet formation closes off and becomes scattering inclusions formed as cylindrical brine tubes, smaller ellipsoidal brine pockets, and very small spherical brine bubbles [Light et al. 2004]. Let $\ell$ denote the length of the non-spherical brine inclusions. For $\ell<0.03 \mathrm{~mm}$ the brine inclusions are spherical (bubbles), but for $0.03 \mathrm{~mm}<\ell<0.5 \mathrm{~mm}$ the brine particles are shaped as prolate ellipsoids (pockets) and for $0.5 \mathrm{~mm} \leq \ell \leq 14.6 \mathrm{~mm}$ they are circular cylinders (tubes) [Light et al. 2003]. The length-todiameter ratio of the brine particles follows the power law [Light et al. 2003]:

$$
\gamma(\ell)=\left\{\begin{array}{ll}
1 & \text { for } \ell \leq 0.03 \mathrm{~mm} \\
10.3 \ell^{0.67} & \text { otherwise }
\end{array} .\right.
$$

All three types of brine particles are distributed according to the power law in Appendix A with the exponent $\alpha=1.96$ where $\ell$ is given in $\mathrm{mm}$. Brine bubbles account for $2 \%$ of the total brine volume, pockets for $6 \%$, and tubes for the remaining $92 \%$ [Light et al. 2003]. This and the equations in Appendix B makes it possible to compute an equivalent number density distribution of spheres for each brine particle type. Finally the relation between volume fraction and number density (28) can be employed to replace the unknown factor in the distributions by

$$
N_{*}=\frac{v}{\frac{4 \pi}{3} \int_{r_{\min }}^{r_{\max }} r_{\mathrm{eq}}(\ell) N_{\mathrm{eq}}(\ell) \mathrm{d} \ell} .
$$

Different types of ice can be constructed by different choices of air and brine volume fractions, and minerals and algae can be included in the same way as they were included in water in Section 4.2 (particle sizes of algae and minerals may need adjustment to larger sizes in ice as compared to water). As examples, we have found optical properties of ice resulting from compacted snow and white first-year ice. Renderings of these two types of ice as well as pure ice for comparison, are presented in Figure 4. The contents of brine and air in compacted snow are $v_{\text {brine }}=8.0 \cdot 10^{-4}$ and $v_{\text {air }}=2.3 \cdot 10^{-4}$.

The importance of using volume-to-area equivalent spheres (see Sec. 2.3) is very significant. At $-15^{\circ} \mathrm{C}$ Light et al. [2004] measured a reduced scattering coefficient of $\sigma_{s}^{\prime} \approx 8$ in first-year sea-ice 


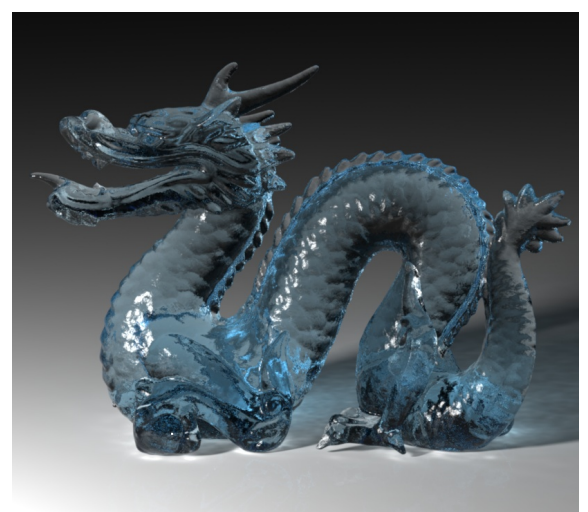

Pure ice

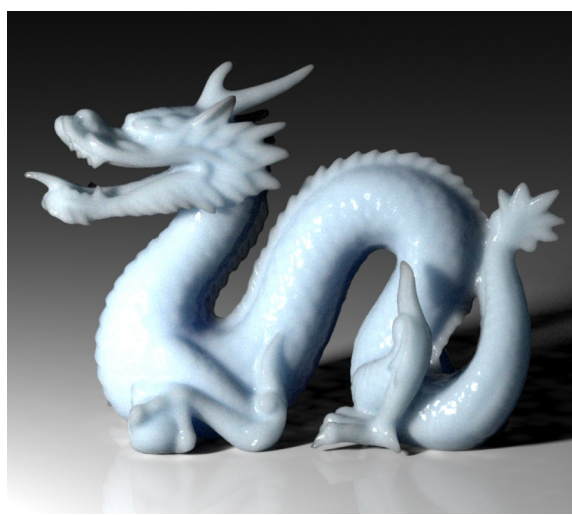

Compacted snow

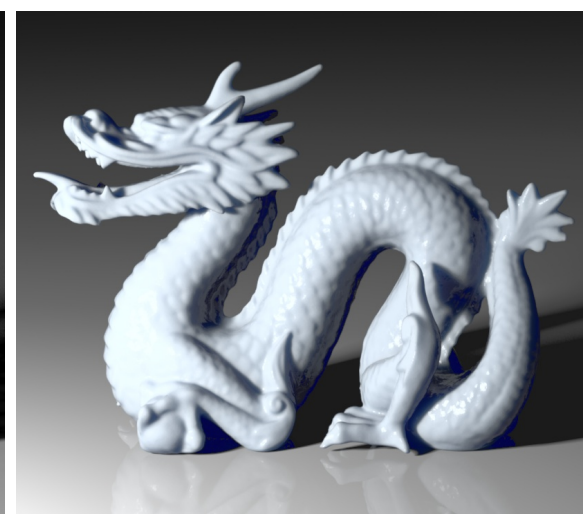

White ice

Figure 4: The Stanford dragon model rendered using different types of ice. The dragon is 50 meters long and at this size it shows the effect of absorption by the ice. This absorption is the reason for the blue light transmitted through the pure ice, the cold blue light of the compacted snow and the deep blue light in the shadow regions of the white ice dragon.

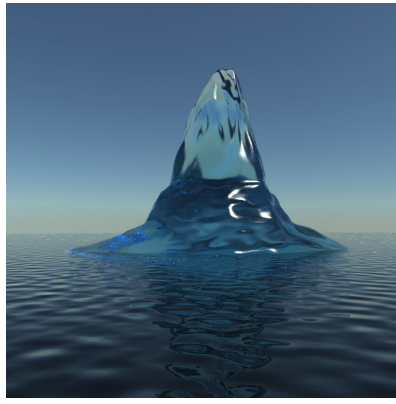

$10 \mathrm{am}$

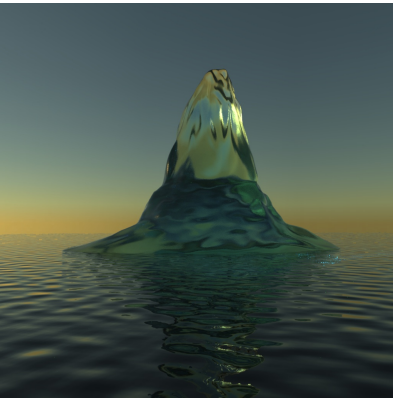

$7 \mathrm{pm}$

Figure 5: A blue iceberg at noon turns green in the evening. These bottle green icebergs are one of nature's peculiarities. We simulated the properties of the green iceberg shown in these images by including a small amount of minerals and only very little air and brine in the ice. The atmospheric lighting model uses Rayleigh scattering to obtain the spectral radiance for the skylight and the sun.

samples with volume fractions $v_{\text {air }}=0.006$ and $v_{\text {brine }}=0.02$. If the shape of the brine inclusions is ignored and all brine is included as bubbles, we get a significant over-estimation, $\sigma_{s}^{\prime} \approx 30$. If volume equivalent spheres are employed, the reduced scattering coefficient is underestimated as $\sigma_{s}^{\prime} \approx 6.4$. The volume-to-area equivalent spheres are a much better choice giving $\sigma_{s}^{\prime} \approx 8.1$.

One of natures peculiar phenomena is bottle green icebergs. This type of iceberg is almost free of brine and air inclusions [Dieckmann et al. 1987; Warren et al. 1993]. They do, however, contain some gray scattering inclusions that we model as minerals. To account for these minerals in the ice, we change the exponent used with the number density distribution in the ocean water case to $\alpha=1.5$. We then include a tiny fraction of brine and a very small fraction of air, as well as a volume fraction of minerals which is $v_{\text {mineral }}=1.5 \cdot 10^{-7}$. A theory for the bottle green sometimes observed in these very clean icebergs, is that their color depends on the light incident through the atmosphere [Lee 1990]. Therefore the ice will look clean and very transmissive during the day, but when sun begins to set, and the spectrum of the incident light shifts towards red wavelengths, the minerals in the iceberg shift the spectrum such that the ice gets a dark green appearance. The rendered bottle green iceberg shown in Figure 5 illustrates the very different appearance of this type of iceberg at two different times of the day.

\section{Conclusion and Future Work}

We have presented a novel generalization of the Lorenz-Mie theory for computing the scattering properties of participating media. Our generalization includes a set of robust recurrence formulas that makes it possible to compute the scattering amplitudes of particles within an absorbing host medium. We have shown how to handle non-spherical particles, and we have presented a framework for computing the bulk optical properties for a given participating medium based on its particle contents.

The generalized Lorenz-Mie theory makes it possible to compute optical properties for the large class of materials composed of particles (possibly non-spherical) in an absorbing host. Previously in graphics it has only been possible to obtain optical properties for such materials through measurements. Our theoretical approach offers a number of advantages in comparison to measurement-based approaches since it can compute all optical properties of a medium at any given wavelength, and it can handle materials such as ice which cannot be measured using existing techniques in graphics.

To demonstrate the theory, we have presented a novel appearance model for milk parameterized by the fat and protein contents. Our appearance model can predict previous measured values and it allows us to estimate the fat and protein contents of milk for which optical properties have been measured. We have also computed optical properties for different types of ocean water and ice. These examples demonstrate the importance of taking an absorbing host medium and non-spherical particles into account.

In the future, we would like to use the theory to estimate the contents of measured materials such as those by Narasimhan et al. [2006]. Such an approach could have interesting perspectives with respect to food and material sciences. It would also be interesting to generalize the theory further in order to support coated and multi-layered particles such as soot within the host medium.

\section{Acknowledgements}

This research was supported by the Otto Mønsted foundation, the V. Kann Rasmussen foundation, AEG Elektronfonden, and CalIT ${ }^{2}$.

\section{References}

Attaie, R., AND Richtert, R. L. 2000. Size distribution of fat globules in goat milk. Journal of Dairy Science 83, 940-944.

Babin, M., Morel, A., Fell, V. F.-S. F., and Stramski, D. 2003. Light scattering properties of marine particles in coastal and open ocean waters as related to the particle mass concentration. Limnology and Oceanography 48, 2, 843-859. 
Babin, M., Stramski, D., Ferrari, G. M., Claustre, H., Bricaud, A., Obolensky, G., AND HoepfFner, N. 2003. Variations in the light absorption coefficients of phytoplankton, nonalgal particles, and dissolved organic matter in coastal waters around Europe. Journal of Geophysical Research 108, C7,3211 (July), 4-1-20.

Bohren, C. F., AND Gilra, D. P. 1979. Extinction by a spherical particle in an absorbing medium. Journal of Colloid and Interface Science 72, 2 (November), 215-221.

Bohren, C. F., And Huffman, D. R. 1983. Absorption and Scattering of Light by Small Particles. John Wiley \& Sons, Inc.

Born, M., AND Wolf, E. 1999. Principles of Optics: Electromagnetic Theory of Propagation, Interference and Diffraction of Light, seventh (expanded) ed. Cambridge University Press.

Bricaud, A., Babin, M., Morel, A., And Claustre, H. 1995. Variability in the chlorophyll-specific absorption coefficients of natural phytoplankton: Analysis and parameterization. Journal of Geophysical Research 100, C7 (July), 13321-13332.

Cachorro, V. E., ANd Salcedo, L. L. 1991. New improvements for Mie scattering calculations. Journal of Electromagnetic Waves and Applications 5, 9, 913-926.

CAllet, P. 1996. Pertinent data for modelling pigmented materials in realistic rendering. Computer Graphics Forum 15, 2, 119-127.

CHANDRASEKHAR, S. 1950. Radiative Transfer. Oxford, Clarendon Press. Unabridged and slightly revised version published by Dover Publications, Inc. in 1960.

Crofcheck, C. L., Payne, F. A., And Mengü C, M. P. 2002. Characterization of milk properties with a radiative transfer model. Applied Optics 41, 10 (April), 2028-2037.

DAVE, J. V. 1969. Scattering of electromagnetic radiation by a large, absorbing sphere. IBM Journal of Research and Development 13, 3 (May), 302-313.

Dieckmann, G., Hemleben, C., And Spindler, M. 1987. Biogenic and mineral inclusions in a green iceberg from the weddell sea, antarctica. Polar Biology 7, 1, 31-33.

Du, H., FuH, R.-C. A., Li, J., CORKAn, L. A., AND Lindsey, J. S. 1998. PhotochemCAD: A computer-aided design and research tool in photochemistry. Photochemistry and Photobiology $68,2,141-142$.

Fox, P. F., And McSweeney, P. L. H. 1998. Dairy Chemistry and Biochemistry. Blackie Academic \& Professional, London.

FU, Q., AND SUN, W. 2006. Apparent optical properties of spherical particles in absorbing medium. Journal of Quantitative Spectroscopy and Radiative Transfer 100, 1-3, 137-142.

GRAY, D. E., Ed. 1972. American Institute of Physics Handbook, 3rd ed. McGraw-Hill.

Grenfell, T. C., AND Perovich, D. K. 1981. Radiation absorption coefficients of polycrystalline ice from 400-1400 nm. Journal of Geophysical Research 86, C8 (August), 7447-7450.

GRENFEll, T. C., AND WARREN, S. G. 1999. Representation of a nonspherical ice particle by a collection of independent spheres for scattering and absorption of radiation. Journal of Geophysical Research 104, D24 (December), 31,697-31,709.

Grenfell, T. C., Neshyba, S. P., ANd Warren, S. G. 2005. Representation of a non-spherical ice particle by a collection of independent spheres for scattering and absorption of radiation: 3. Hollow columns and plates. Journal of Geophysical Research 110, D17203 (August), 1-15.

Grenfell, T. C. 1983. A theoretical model of the optical properties of sea ice in the visible and near infrared. Journal of Geophysical Research 88, C14 (November), 9723-9735.

HAle, G. M., AND Querry, M. R. 1973. Optical constants of water in the 200-nm to 200- $\mu \mathrm{m}$ wavelength region. Applied Optics 12, 3 (March), 555-563.
Hawkins, T., Einarsson, P., And Debevec, P. 2005. Acquisition of time-varying participating media. Proceedings of ACM SIGGRAPH 2005 24, 3, 812-815.

Henyey, L. G., AND GReEnstein, J. L. 1940. Diffuse radiation in the galaxy. Annales d'Astrophysique 3,117-137. Also in The Astrophysical Journal 93, 1941.

JACKÈL, D., AND WALTER, B. 1997. Modeling and rendering of the atmosphere using Mie-scattering. Computer Graphics Forum $16,4,201-210$.

Jensen, H. W., Marschner, S. R., Levoy, M., And HanraHAN, P. 2001. A practical model for subsurface light transport. In Proceedings of SIGGRAPH 2001, 511-518.

KatTawar, G. W., AND Plass, G. N. 1967. Electromagnetic scattering from absorbing spheres. Applied Optics 6, 8 (August), 1377-1382.

LEE, JR., R. L. 1990. Green icebergs and remote sensing. Journal of Optical Society America A 7, 10 (October), 1862-1874.

LIDE, D. R., Ed. 2006. CRC Handbook of Chemistry and Physics, 87th ed. CRC Press.

Light, B., MAYKut, G. A., AND GRenfell, T. C. 2003. Effects of temperature on the microstructure of first-year arctic sea ice. Journal of Geophysical Research 108, C2, 3051 (February), 33$1-16$.

Light, B., Maykut, G. A., And Grenfell, T. C. 2004. A temperature-dependent, structural-optical model of first-year sea ice. Journal of Geophysical Research 109, C06013 (June), 1-16.

LORENZ, L. 1890. Lysbevægelser i og uden for en af plane Lysbølger belyst Kugle. Det kongelig danske Videnskabernes Selskabs Skrifter, 2-62. 6. Række, Naturvidenskabelig og Mathematisk Afdeling VI, 1.

Mackowski, D. W., Altenkirch, R. A., And Menguc, M. P. 1990. Internal absorption cross sections in a stratified sphere. Applied Optics 29, 10 (April), 1551-1559.

Michalski, M.-C., BRIARD, V., AND MiChel, F. 2001. Optical parameters of milk fat globules for laser light scattering measurements. Lait 81, 787-796.

MIE, G. 1908. Beiträge zur Optik trüber Medien, speziell kolloidaler Metallösungen. Annalen der Physik 25, 3, 377-445. IV. Folge.

Mundy, W. C., Roux, J. A., And Smith, A. M. 1974. Mie scattering by spheres in an absorbing medium. Journal of the Optical Society of America 64, 12 (December), 1593-1597.

NARAsimhan, S. G., Gupta, M., Donner, C., RAMAmoorTHI, R., NAYAR, S. K., AND Jensen, H. W. 2006. Acquiring scattering properties of participating media by dilution. ACM Transactions on Graphics (Proceedings of SIGGRAPH 2006) 25, 3 (July), 1003-1012.

Neshyba, S. P., Grenfell, T. C., And Warren, S. G. 2003. Representation of a non-spherical ice particle by a collection of independent spheres for scattering and absorption of radiation: 2 . Hexagonal columns and plates. Journal of Geophysical Research 108, D15, 4448 (August), 6-1-18.

Olson, D. W., White, C. H., And Richter, R. L. 2004. Effect of pressure and fat content on particle sizes in microfluidized milk. Journal of Dairy Science 87, 10, 3217-3223.

PALIK, E. D., Ed. 1985. Handbook of Optical Constants of Solids. Academic Press.

Pegau, W. S., Gray, D., And Zaneveld, J. R. V. 1997. Absorption and attenuation of visible and near-infrared light in water: Dependence on temperature and salinity. Applied Optics 36, 24 (August), 6035-6046.

Pope, R. M., AND FRY, E. S. 1997. Absorption spectrum (380$700 \mathrm{~nm}$ ) of pure water. ii. integrating cavity measurements. Applied Optics 36, 33 (November), 8710-8723. 
RandrianalisoA, J., Baillis, D., And Pilon, L. 2006. Modeling radiation characteristics of semitransparent media containing bubbles or particles. Journal of the Optical Society of America A 23, 7 (July), 1645-1656.

Riley, K., Ebert, D. S., Kraus, M., Tessendorf, J., And HANSEN, C. 2004. Efficient rendering of atmospheric phenomena. In Proceedings of Eurographics Symposium on Rendering 2004, H. W. Jensen and A. Keller, Eds., 375-386.

RUSHMEIER, H. 1995. Input for participating media. In Realistic Input for Realistic Images, ACM SIGGRAPH '95 Course Notes. Also appeared in the ACM SIGGRAPH '98 Course Notes - A Basic Guide to Global Illumination.

Schmidt, D. G., Walstra, P., And Buchheim, W. 1973. The size distribution of casein micelles in cow's milk. Netherland's Milk Dairy Journal 27, 128-142.

Stockman, A., AND Sharpe, L. T. 2000. The spectral sensitivities of the middle- and long-wavelength-sensitive cones derived from measurements in observers of known genotype. Vision Research 40, 13, 1711-1737.

Tong, X., Wang, J., Lin, S., Guo, B., And Shum, H. 2005. Modeling and rendering of quasi-homogeneous materials. Proceedings of ACM SIGGRAPH 2005 24, 3, 1054-1061.

VAN DE HULST, H. C. 1949. On the attenuation of plane waves by obstacles of arbitrary size and form. Physica 15, 8-9 (September), 740-746.

VAN DE HUlst, H. C. 1957,1981. Light Scattering by Small Particles. Dover Publications, Inc., New York. Unabridged and corrected republication of the work originally published in 1957.

VideEn, G., AND Sun, W. 2003. Yet another look at light scattering from particles in absorbing media. Applied Optics 42, 33 (November), 6724-6727.

Walstra, P., And Jenness, R. 1984. Dairy Chemistry and Physics. John Wiley \& Sons, New York.

WALstra, P. 1975. Effect of homogenization on the fat globule size distribution in milk. Netherland's Milk Dairy Journal 29, 279-294.

Warren, S. G., Roesler, C. S., Morgan, V. I., Brandt, R. E., Goodwin, I. D., And Allison, I. 1993. Green icebergs formed by freezing of organic-rich seawater to the base of antarctic ice shelves. Journal of Geophysical Research 98, C4 (April), 6921-6928.

WARren, S. G., BRANDT, R. E., AND GREnfell, T. C. 2006. Visible and near-ultraviolet absorption spectrum of ice from transmission of solar radiation into snow. Applied Optics 45, 21 (July), 5320-5334.

WARREN, S. G. 1984. Optical constants of ice from the ultraviolet to the microwave. Applied Optics 23, 8 (April), 1206-1225.

WiscomBE, W. J. 1980. Improved Mie scattering algorithms. Applied Optics 19, 9 (May), 1505-1509.

WU, Z. S., AND WANG, Y. P. 1991. Electromagnetic scattering for multilayered sphere: Recursive algorithms. Radio Science 26, 6, 1393-1401.

Wyman, D. R., Patterson, M. S., And Wilson, B. C. 1989. Similarity relations for the interaction parameters in radiation transport. Applied Optics 28 (December), 5243-5249.

YAng, P., GaO, B.-C., Wiscombe, W. J., Mischenko, M. I., Platnick, S. E., HuAng, H.-L., Baum, B. A., HU, Y. X., Winker, D. M., TsAY, S.-C., AND PARK, S. K. 2002. Inherent and apparent scattering properties of coated or uncoated spheres embedded in an absorbing host medium. Applied Optics 41, 15 (May), 2740-2758.

YANG, W. 2003. Improved recursive algorithm for light scattering by a multilayered sphere. Applied Optics 42, 9 (March), 17101720.
YIN, J., AND PILON, L. 2006. Efficiency factors and radiation characteristics of spherical scatterers in an absorbing medium. Journal of the Optical Society of America A 23, 11 (November), 2784-2796.

\section{A Common Size Distributions}

The log-normal distribution with mean value $\mu$ and standard deviation $\sigma$ is as follows (for a particle volume frequency)

$$
r^{3} N(r)=\frac{1}{r \beta \sqrt{2 \pi}} e^{-\frac{1}{2}\left(\frac{\ln r-\alpha}{\beta}\right)^{2}},
$$

where $r$ is the particle radius and

$$
\alpha=\ln \mu-\frac{1}{2} \ln \left(\frac{\sigma^{2}}{\mu^{2}}+1\right) \quad, \quad \beta=\sqrt{\ln \left(\frac{\sigma^{2}}{\mu^{2}}+1\right)} .
$$

The power law distribution is $N(r)=N_{*} r^{-\alpha}$, where $\alpha$ is usually determined empirically and $N_{*}$ is a constant which can be determined by the relationship between number density and volume fraction (28).

\section{B Non-Spherical Particle Formulas}

We present analytic formulas for two common non-spherical particle shapes found in scattering media.

\section{B.1 Ellipsoids}

Given a particle with length $\ell$ and length-to-diameter ratio $\gamma$. The surface area and volume of a prolate ellipsoid are:

$$
A_{\mathrm{el}}=\frac{\pi}{2} \frac{\ell^{2}}{\gamma^{2}}\left(1+\gamma \frac{\sin ^{-1} \varepsilon}{\varepsilon}\right) \quad \text { and } \quad V_{\mathrm{el}}=\frac{\pi}{6} \frac{\ell^{3}}{\gamma^{2}}
$$

where $\varepsilon=\sqrt{1-1 / \gamma^{2}}$ is the ellipse eccentricity. The radius of the volume-to-area equivalent sphere is

$$
r_{\mathrm{eq}, \mathrm{el}}=\frac{\ell \varepsilon}{\varepsilon+\gamma \sin ^{-1} \varepsilon} .
$$

\section{B.2 Cylinders}

\begin{tabular}{|c|c|c|c|c|c|c|c|}
\hline $\begin{array}{r}\lambda_{0} \\
{[\mathrm{~nm}]}\end{array}$ & water $^{1}$ & brine $^{2}$ & $\mathrm{fat}^{3}$ & $\begin{array}{r}\operatorname{Im}(\eta) \\
\text { water }+B 2^{4}\end{array}$ & mineral $^{5}$ & algae $^{6}$ & ice $^{7}$ \\
\hline 375 & $3.39 e-10$ & $4.59 e-10$ & $4.0 e-6$ & $2.93 e-7$ & $2.12 e-3$ & $1.84 e-4$ & $2.42 e-11$ \\
\hline 400 & $2.11 e-10$ & $3.38 e-10$ & $6.4 e-6$ & $2.60 e-7$ & $1.66 e-3$ & $1.96 e-4$ & $2.37 e-11$ \\
\hline 25 & 1.62 & 2.23 & 8.6 & 3.36 & 1.30 & 2.89 & 3.52 \\
\hline 50 & 3.30 & 3.05 & $1.1 e-5$ & 4.10 & 1.01 & 3.11 & 9.24 \\
\hline 75 & 4.31 & 4.04 & 1.1 & 3.33 & $7.84 e-4$ & 2.79 & $2.38 e-10$ \\
\hline 500 & $8.12 e-10$ & $7.76 e-10$ & $1.0 e-5$ & $1.08 e-7$ & $6.07 e-4$ & $2.14 e-4$ & $5.89 e-10$ \\
\hline 25 & $1.74 e-9$ & $1.69 e-9$ & $4.7 e-6$ & $5.64 e-8$ & 4.59 & 1.26 & $1.24 e-9$ \\
\hline 50 & 2.47 & 2.42 & 4.6 & 6.02 & 3.61 & $8.19 e-5$ & 2.29 \\
\hline 75 & 3.53 & 3.48 & 4.7 & 7.91 & 2.77 & 5.57 & 3.80 \\
\hline 600 & $1.06 e-8$ & $1.05 e-8$ & $4.9 e-6$ & $7.95 e-8$ & $2.13 e-4$ & $6.03 e-5$ & $5.73 e-9$ \\
\hline 25 & 1.41 & 1.40 & 5.0 & 8.58 & 1.63 & 7.86 & 9.50 \\
\hline 50 & 1.76 & 1.76 & 5.0 & 9.32 & 1.25 & $1.00 e-4$ & $1.43 e-8$ \\
\hline 75 & 2.41 & 2.40 & 5.1 & 7.37 & $9.52 e-5$ & 2.53 & \\
\hline 700 & $3.48 e-8$ & $3.44 e-8$ & $5.2 e-6$ & $1.14 e-7$ & $7.26 e-5$ & $3.91 e-5$ & $2.90 e-8$ \\
\hline 25 & 8.59 & 8.51 & 5.2 & 1.33 & 5.53 & 3.91 & 4.17 \\
\hline 50 & $1.47 e-7$ & $1.47 e-7$ & 5.2 & $\begin{array}{l}1.00 \\
2.20\end{array}$ & 4.20 & 3.91 & 5.87 \\
\hline 775 & $1.49 e-7$ & $1.49 e-7$ & $5.2 e-6$ & $2.35 e-7$ & $3.19 e-5$ & $3.91 e-5$ & $9.37 e-8$ \\
\hline \\
\hline \multicolumn{8}{|c|}{$\begin{array}{l}\text { In this table, we use the notation } x e-y=x .100 \\
{ }^{1} \text { Data from Pope and Fry [1997] for } 380-700 \mathrm{~nm} \text {. Remaining from Hale and Query [1973]. }\end{array}$} \\
\hline \multicolumn{8}{|c|}{$\begin{array}{l}{ }^{1} \text { Data from Pope and Fry [1997] for } 380-700 \mathrm{~nm} \text {. Remaining from Hale and Query [1973]. } \\
{ }^{2} \text { Data for pure water }{ }^{1} \text { corrected using slopes from Pegau et al. [1997]. }\end{array}$} \\
\hline \multicolumn{8}{|c|}{${ }^{3}$ Data from Michalski et al. [2001]. } \\
\hline \multicolumn{8}{|c|}{ 4 Data from the PhotochemCAD application [Du et al. 1998]. } \\
\hline \multicolumn{8}{|c|}{$\begin{array}{l}\left.{ }^{5} \text { Data from the PhotochemCAD application [Du et al. } 1998\right] \text {. } \\
\text { Example spectrum computed using results from Babin et al. [2003b]. }\end{array}$} \\
\hline \multicolumn{8}{|c|}{${ }^{6}$ Example spectrum computed using data from Bricaud et al. [1995]. } \\
\hline
\end{tabular}

For a circular cylinder with length $\ell$ and length-to-diameter ratio $\gamma$ :

$$
A_{\mathrm{cy}}=\frac{\pi}{2} \frac{\ell^{2}}{\gamma^{2}}(1+2 \gamma) \quad, \quad V_{\mathrm{cy}}=\frac{\pi}{4} \frac{\ell^{3}}{\gamma^{2}} \quad, \quad r_{\mathrm{eq}, \mathrm{cy}}=\frac{3 \ell}{2+4 \gamma} .
$$

\section{Imaginary Part of Refractive Indices}

